\title{
Erratum to: Axillary Recurrence Rate Following Negative Sentinel Node Biopsy for Invasive Breast Cancer: Long-Term Follow-Up
}

\author{
John V. Kiluk, MD ${ }^{1}$, Quan P. Ly, MD ${ }^{2}$, Alfredo A. Santillan, MD, MPH ${ }^{3}$, Tammi Meade, BS $^{1,4}$, \\ Daniel Ramos, $\mathbf{B S}^{1,4}$, Douglas S. Reintgen, $\mathrm{MD}^{5}$, Sophie Dessureault, MD, $\mathrm{PhD}^{1}$, Michelle Davis, $\mathrm{BS}^{1}$, \\ Corinne Shamehdi, $\mathrm{BS}^{1}$, and Charles E. Cox, $\mathrm{MD}^{1,4,6}$
}

${ }^{1}$ H. Lee Moffitt Cancer Center and Research Institute (MCC), Tampa, FL; ${ }^{2}$ University of Nebraska Medical Center, Omaha, NE; ${ }^{3}$ Division of Surgical Oncology, University of Texas Health Science Center at San Antonio, San Antonio, TX; ${ }^{4}$ Department of Surgery, USF College of Medicine, Tampa, FL; ${ }^{5}$ Lakeland Regional Cancer Center, Lakeland, FL; ${ }^{6}$ USF Breast Health, Carol and Frank Morsani Center for Advanced Health Care, Tampa, FL

\begin{abstract}
Objective. Sentinel lymph node (SLN) biopsy has replaced axillary lymph node dissection (ALND) as the staging procedure for breast cancer. SLN biopsy causes less morbidity and is more cost effective than complete ALND. Lymphatic mapping and SLN biopsy have a low false-negative rate, but long-term outcomes in large consecutive series of patients are unavailable.

Methods. Retrospective review of a prospectively accrued institutional breast cancer database was performed. The initial mapping of 1,528 patients with invasive breast cancer that demonstrated negative sentinel node biopsy and no axillary dissection in 1,530 cases between January 1995 and June 2003 were collated and reviewed to achieve a long-term follow-up. These 1,528 patients were reviewed for follow-up time, local recurrences, distant metastases, and survival.

Results. A total of 1,530 consecutively mapped invasive breast cancer cases had negative SLN biopsy and no ALND. The mean invasive tumor size of was $1.40 \mathrm{~cm}$. Of patients, 1,212 (79.2\%) underwent lumpectomy and 318
\end{abstract}

The online version of the original article can be found under doi: 10.1245/s10434-009-0704-1.

An earlier version of this manuscript was published. The revised, final version of this article follows in its entirety.

(C) Society of Surgical Oncology 2009

Published Online: 3 December 2009

C. E. Cox, MD

e-mail: coxce@hotmail.com
(20.8\%) underwent mastectomy. Median follow-up was 63 months (range 0.1-144 months). There have been 4 $(0.26 \%)$ cases presenting with local axillary recurrences, $54(3.53 \%)$ cases presenting with local recurrences in the ipsilateral breast/chest wall, and $24(1.57 \%)$ cases presenting with distant metastases.

Conclusion. These data confirm that SLN biopsy is an effective and safe alternative to ALND for detection of nodal metastases in patients with invasive breast cancer and validates its use as the standard tool for nodal staging.

The status of the axillary nodal basin is one of the most important prognostic indicators for recurrence and survival in patients with breast cancer. ${ }^{1,2}$ Until the introduction of sentinel node mapping in the early $1990 \mathrm{~s}$, the standard operation for staging the axilla involved level I and II axillary nodal dissection. This operation provided maximum local control of cancer while providing valuable staging information that guided additional treatment choices and provided prognostic information for patients and clinicians alike. Unfortunately, the advantages of axillary dissection did not come without significant morbidity. Acute and chronic lymphedema, paresthesia and pain from intercostal and intercostal-brachial nerve injury, and seromas from axillary dissections made the ramifications of this surgery some of the most significant complaints relating to breast cancer treatment. ${ }^{3}$

As screening mammography and breast cancer awareness increased, the percentage of patients with positive lymph nodes decreased to approximately $30 \% .{ }^{4}$ As a result, $70 \%$ of patients with breast cancer were taking on the 
morbidity of axillary dissection while receiving minimal benefit. The advent of lymphatic mapping, which removes the first few nodes that drain the breast, provided a solution to this problem that minimized morbidity from axillary surgery while improving the ability to stage the axilla accurately. ${ }^{5,6}$

Initially, sentinel node biopsies were validated with mandatory completion axillary nodal dissections following removal of the sentinel node to ensure a low rate of falsenegative nodes being missed. Multiple published trials reported false-negative rates of 5-10\%, which was deemed acceptable to avoid axillary dissection with a negative sentinel node. ${ }^{7-9}$ Further studies demonstrated that this false-negative rate could be lowered to less than $5 \%$ with immunohistochemical staining of the sentinel node and increased surgeon experience. ${ }^{8}$ As a result, the accuracy of a sentinel node biopsy improved the detection of locating axillary metastases, where the single node could be thoroughly sectioned, immunohistochemically (IHC) stained, and reviewed pathologically versus the prior cursory review of hematoxylin and eosin (H\&E) stains of single sections on all bivalved harvested nodes. ${ }^{10}$

While these initial studies have been promising, followup data have been limited to a few years following surgery. While diagnostic accuracy may have improved with sentinel node biopsy, local recurrence should be improved. The purpose of this study is to demonstrate the accuracy of sentinel node biopsy alone without combined axillary dissection as an effective tool for axillary staging and concomitant local control by demonstrating a low axillary recurrence rate on long-term follow-up.

\section{MATERIALS AND METHODS}

An Institutional Review Board (IRB)-approved, Health Insurance Portability and Accountability Act (HIPPA)compliant breast cancer database and electronic health record (IRB\# 102554) prospectively accrued 3,682 patients undergoing 4,186 sentinel node biopsies between January 1995 and June 2003 at the Moffitt Cancer Center. Under separate IRB approval (IRB\# 105928), this database was queried for invasive breast cancer patients with pathology reports showing no metastatic disease in the sentinel node and no axillary further axillary dissection.

A total of 1,530 sentinel node biopsies performed on 1,528 patients were identified that fulfilled this requirement. The sentinel nodes were identified using a combination technique of technetium sulfur colloid and isosulfan blue dye. ${ }^{11}$ Routine pathologic evaluation included intraoperative imprint cytology of nodes followed by sectioning of the node into 2-mm sections, which were placed sequentially into cassettes, paraffin embedded, faced, cut, and stained with $\mathrm{H} \& \mathrm{E}$, followed by cuts at 50 and 100 microns stained for CK-IHC (CAM 5.2; Becton Dickinson) and, as a control for the IHC stains, counterstained with hematoxylin. All patients had a negative sentinel node by both H\&E and cytokeratin staining (NOi-).

Follow-up data was obtained from an IRB-approved (IRB \#102554) breast cancer database and chart review that details follow-up at our institution. To improve the accuracy of survival data for all patients, data was checked against the Social Security Death Index online database (http://ssdi.rootsweb.com). Statistical analysis was performed by Wilcoxon rank-sum test, chi-square test, Fisher's exact test, Kruskal-Wallis, and analysis of variance (ANOVA), when appropriate. A two-tailed $P$ value of $<0.05$ was considered statistically significant. Statistical analyses were performed using Stata 9.0 software (StataCorp, College Station, TX).

\section{RESULTS}

Between January 1995 and June 2003, 1,530 cases of invasive breast cancer were found to have a sentinel node negative for malignancy and did not receive axillary dissection. The mean age at time of surgery was 60 years (range 25-91 years). The histology of the invasive tumor was as follows: invasive ductal $(78.0 \%)$, invasive lobular $(9.0 \%)$, mixed $(5.2 \%)$, tubular $(3.4 \%)$, mucinous $(1.7 \%)$, other $(2.8 \%)$. The mean size of the invasive tumor was $1.4 \mathrm{~cm}$ (range $0.1-14.1 \mathrm{~cm}$ ). Tumor histology revealed high-grade tumor in 381 cases $(25 \%)$ and lymphovascular invasion in only 34 cases $(0.02 \%)$.

The median and mean number of SLNs excised per axilla were 2 (range 1-12) and 2.4, respectively. All sentinel nodes removed were negative for malignancy by hematoxylin and eosin as well as immunohistochemistry. The median and mean number of nonsentinel nodes excised were 0 (range 0-9) and 0.9, respectively. All nonsentinel nodes were negative for hematoxylin and eosin only. The median and mean number of total lymph nodes removed were 3 (range 1-16) and 3.4, respectively. Fortytwo patients had ten more total nodes removed. While these patients obviously had more extensive sampling of the axilla, formal axillary dissection was still not performed and axillary tissue remained in the axilla of these patients. A total of 1,214 cases $(79 \%)$ had their primary breast cancer resected with a lumpectomy, whereas 316 cases $(21 \%)$ involved mastectomy. Adjuvant chemotherapy was delivered in $392(25.6 \%)$ patients, whereas 780 $(51.0 \%)$ received hormonal therapy following their operation for breast cancer.

The median time of follow-up for the entire cohort of 1,530 cases was 63 months (range 0.1-144 months). The clinicopathologic and follow-up characteristics by site of recurrence are shown in Tables 1 and 2. No local, regional 
or distant recurrence was identified in 1,429 cases. Four patients $(0.26 \%)$ have presented with local axillary recurrences. The median time to axillary recurrence was 73 months (range 23-108) after initial sentinel lymph node operation. These four cases had their initial surgery between 1998 and 2001, with 290 cases being performed prior to the first case with axillary recurrence. One of these four patients developed an axillary recurrence at the same time as an ipsilateral chest wall recurrence was identified. All four patients received axillary dissection for their recurrence, with one having neoadjuvant chemotherapy prior to surgery. The median number of positive lymph nodes found at the time of axillary dissection was 1.5 nodes (range 1-3). Two of these patients died of metastatic disease (liver and lung) while one died with cardiac and renal problems. We compared clinical and pathological characteristics between patients with local axillary recurrences and those with no recurrence, and found a higher proportion of patients with high histologic grade among those with local axillary recurrence $(75 \%$ versus $27 \%$, $P=0.03)$. No significant difference in age, tumor size, rate of lymphovascular invasion, type of surgery or number of SLN or non-SLN removed was identified.

Twenty-four patients (1.57\%) presented with distant metastatic disease, a median of 48 months (range 4128 months) after their sentinel node operation. The patients within this group had significantly higher median tumor size when compared with patients with no recurrence $(1.8$ versus $1.2 \mathrm{~cm}, P<0.01)$. Furthermore, a significant proportion of these patients were found to have high-grade tumors compared with those patients with no recurrence $(52 \%$ versus $27 \%, P=0.02)$. Of note, patients with distant metastatic disease had the lowest rate of hormonal therapy (25\%). There were 54 cases of recurrence in the ipsilateral breast $(3.5 \%)$ following initial surgery after a median time of 48 months (range 4-128 months). There were 19 patients $(1.24 \%)$ who had a new cancer develop in the contralateral breast after a median time of 27 months (range 7-82 months). There have been 157 patients $(10.3 \%)$ who have died after a median time of 50 months (range 0.1-122 months). Patients with axillary recurrence (75\%) and distant recurrence $(67 \%)$ were found to have higher overall mortality compared with those with no recurrence $(8 \%)$, ipsilateral breast $(19 \%)$, and contralateral breast $(16 \%)$ recurrence.

\section{DISCUSSION}

As surgeon experience with sentinel node biopsies has improved and mandatory axillary dissections have been abandoned, the efficacy of negative sentinel node biopsy needs long-term validation. Not only does sentinel node biopsy need to accurately stage the patient's cancer, but it must also provide local control of disease comparable to

TABLE 1 Clinicopathologic characteristics by site of recurrence

\begin{tabular}{|c|c|c|c|c|c|c|}
\hline & $\begin{array}{l}\text { No recurrence } \\
(n=1,429)\end{array}$ & $\begin{array}{l}\text { Ipsilateral axilla } \\
(n=4)\end{array}$ & $\begin{array}{l}\text { Ipsilateral breast/chest } \\
\text { wall }(n=54)\end{array}$ & $\begin{array}{l}\text { Contralateral breast } \\
(n=19)\end{array}$ & $\begin{array}{l}\text { Metastatic disease } \\
(n=24)\end{array}$ & $P$-Value \\
\hline Median age, years (range) & $60(25-91)$ & $65(44-89)$ & $49(25-80)$ & $72(41-85)$ & $56(37-80)$ & $<0.01$ \\
\hline \multicolumn{7}{|l|}{ Surgery $(\%)$} \\
\hline Mastectomy & $299(21)$ & $1(25)$ & $7(13)$ & $6(32)$ & $5(21)$ & \multirow[t]{2}{*}{0.66} \\
\hline Lumpectomy & $1,130(79)$ & $3(75)$ & $47(87)$ & $13(68)$ & $19(79)$ & \\
\hline Median tumor size, cm (range) & $1.2(0.1-4.2)$ & $1.5(1.5-2.1)$ & $1.5(0.1-3.5)$ & $1.4(0.3-2.5)$ & $1.8(0.8-3.5)$ & $<0.01$ \\
\hline \multicolumn{7}{|l|}{ Histologic grade $(\%)$} \\
\hline Low/moderate & $925(73)$ & $1(25)$ & $23(53)$ & $15(83)$ & $10(48)$ & \multirow[t]{2}{*}{$<0.01$} \\
\hline High & $343(27)$ & $3(75)$ & $21(47)$ & $3(17)$ & $11(52)$ & \\
\hline \multicolumn{7}{|l|}{ Lymphovascular invasion (\%) } \\
\hline Yes & $31(2)$ & $0(0)$ & $2(4)$ & $1(5)$ & $0(0)$ & \multirow[t]{2}{*}{0.43} \\
\hline No & $1386(98)$ & $4(100)$ & $51(96)$ & $18(95)$ & $24(100)$ & \\
\hline \multicolumn{7}{|l|}{ SLN removed } \\
\hline Median (range) & $2(1-12)$ & $3(1-5)$ & $2(1-7)$ & $1(1-4)$ & $1(2-7)$ & 0.22 \\
\hline Mean & 2.45 & 3 & 2.42 & 1.63 & 2.62 & 0.25 \\
\hline \multicolumn{7}{|l|}{ Non-SLN removed } \\
\hline Median (range) & $0(0-9)$ & $0(0-1)$ & $0.5(0-8)$ & $0(0-8)$ & $0.5(0-9)$ & 0.69 \\
\hline Mean & 0.96 & 0.25 & 1.16 & 1.31 & 1.25 & 0.57 \\
\hline \multicolumn{7}{|l|}{ Total LN removed } \\
\hline Median (range) & $3(1-15)$ & $3.5(1-5)$ & $3(1-12)$ & $2(1-11)$ & $3(1-16)$ & 0.39 \\
\hline Mean & 3.42 & 3.25 & 3.59 & 2.94 & 3.87 & 0.76 \\
\hline
\end{tabular}


TABLE 2 Follow-up and treatment characteristics by site of recurrence

\begin{tabular}{|c|c|c|c|c|c|c|}
\hline & $\begin{array}{l}\text { No recurrence } \\
(n=1,429)\end{array}$ & $\begin{array}{l}\text { Ipsilateral axilla } \\
(n=4)\end{array}$ & $\begin{array}{l}\text { Ipsilateral breast/chest } \\
\text { wall }(n=54)\end{array}$ & $\begin{array}{l}\text { Contralateral } \\
\text { breast }(n=19)\end{array}$ & $\begin{array}{l}\text { Metastatic } \\
\text { disease }(n=24)\end{array}$ & $P$-Value \\
\hline Median follow-up, months (range) & $63(0.1-144)$ & $82(41-112)$ & $76(8-129)$ & $65(0.3-112)$ & $57(16-122)$ & 0.02 \\
\hline $\begin{array}{l}\text { Median time to recurrence, } \\
\text { months (range) }\end{array}$ & na & $73(23-108)$ & $48(4-128)$ & $27(7-82)$ & $39(9-131)$ & 0.31 \\
\hline \multicolumn{7}{|l|}{ Chemotherapy (\%) } \\
\hline Yes & $349(27)$ & $2(50)$ & $21(40)$ & $5(26)$ & $15(62)$ & 0.01 \\
\hline No & $958(73)$ & $2(50)$ & $31(60)$ & $14(74)$ & $9(38)$ & \\
\hline \multicolumn{7}{|l|}{ Hormonal therapy $(\%)$} \\
\hline Yes & $736(57)$ & $2(50)$ & $27(52)$ & $9(47)$ & $6(25)$ & 0.02 \\
\hline No & $565(43)$ & $2(50)$ & $25(48)$ & $10(53)$ & $18(75)$ & \\
\hline \multicolumn{7}{|l|}{ Death $(\%)$} \\
\hline Yes & $125(8)$ & $3(75)$ & $10(19)$ & $3(16)$ & $16(67)$ & $<0.01$ \\
\hline No & $1304(92)$ & $1(25)$ & $44(81)$ & $16(84)$ & $8(33)$ & \\
\hline
\end{tabular}

axillary dissection. There is little argument over the accuracy of staging with a sentinel node biopsy; however, the risk of axillary recurrence after a sentinel node biopsy alone is slowly emerging.

The universal convention for reporting sentinel node false-negative (FN) rates throughout the world's literature and the methods for calculating $\mathrm{FN}$ rates has led to great confusion. The following example illustrates the point: 1 FN case out of 173 patients mapped with 120 negative sentinel nodes and 53 positive sentinel nodes constitutes our original data for all patients having a sentinel node biopsy followed by axillary dissection. ${ }^{12}$ The method that has been universally reported in sentinel node papers for the calculation of the false-negative rate is $\mathrm{FN} /$ (true positives $+\mathrm{FN})$, i.e., $1 /(53+1)=1.9 \%$, which defines how many of the positive cases would be missed by a sentinel node mapping. ${ }^{7-9}$ This should be compared with FN/(true negatives $+\mathrm{FN})$, i.e., $1 /(119+1)=0.83 \%$, which was not held as the convention but that defines how many of the node-negative patients would have a positive lymph node following axillary dissection. Interestingly, the latter calculation is the one that most closely parallels the actual long-term follow-up data $(0.26 \%$ ipsilateral recurrence rate) and that is the more clinically relevant. Despite all of these concerns regarding overreporting of false-negative rates for SLN mapping throughout the literature, the observed rate of recurrence in patients with a negative sentinel node biopsy remains lower than any calculated risk, validating its efficacy in staging and local control. With regard to patients presenting with distant disease and without local axillary recurrence following a negative sentinel node biopsy, we are unable to assess whether this was a falsely negative sentinel node, extra-axillary drainage to nodes such as the internal mammary chain, or hematogenous spread of disease. As these tumors had the largest average size of invasion and the lowest rate of receiving hormonal therapy, we believe that the majority of these patients had more aggressive, estrogen-receptornegative tumors.

A review article and meta-analysis was recently published that assessed the existing published data on this subject through the beginning of $2007 .{ }^{13}$ Forty-eight papers were identified with an average follow-up of 34 months. The axillary recurrence rate after a negative sentinel node biopsy was identified as $0.3 \%$, which involved 14,959 patients with most recurrences occurring within 20 months of the negative sentinel node biopsy. The largest study included in the meta-analysis was Memorial Sloan Kettering's 2005 evaluation of 2,340 patients with 31-month follow-up, demonstrating a $0.13 \%$ recurrence rate. ${ }^{14}$ Since this review article was published, the Swedish Multicenter Cohort Study reported a $1.2 \%$ recurrence rate in 2,246 patients with a mean 37-month follow-up. ${ }^{15}$ Table 3 summarizes the recurrence rates of publications with at least 500 patients. $^{14-22}$

An interesting note should be made of the meta-analysis in that only one paper had follow-up longer than 5 years (65 months). ${ }^{23}$ The recurrence rate in this group was higher than in a majority of the papers published in the past $(2.68 \%)$. In the evaluation of our local axillary recurrences, the median time to axillary recurrence was 73 months, which is longer than any published data median follow-up.

Although our data and multiple other publications support an acceptable rate $(0.1-0.3 \%)$ of local axillary recurrences with short-term follow-up, we caution that this rate may increase as the length of follow-up extends past 5 years, as half of our local recurrences occurred after this period of time. However, in response to questioning the local control of a negative sentinel node biopsy, we doubt that the rate of axillary recurrences would rise to levels 
TABLE 3 Publications on axillary recurrence rates following negative sentinel node biopsy with at least 500 patients

\begin{tabular}{|c|c|c|c|c|c|}
\hline First author & Year & Patients & Follow-up (months) & $\begin{array}{l}\text { Axillary } \\
\text { recurrences }\end{array}$ & $\begin{array}{l}\text { Axillary recurrence } \\
\text { rate }(\%)\end{array}$ \\
\hline Blanchard $^{19}$ & 2003 & 685 & 29 (mean) & 1 & 0.15 \\
\hline Naik $^{14}$ & 2004 & 2,340 & 31 (median) & 3 & 0.13 \\
\hline Veronesi ${ }^{17}$ & 2005 & 953 & 38 (median) & 3 & 0.3 \\
\hline Jeruss $^{20}$ & 2005 & 592 & 27 (mean) & 1 & 0.17 \\
\hline Swenson $^{21}$ & 2005 & 580 & 33 (median) & 3 & 0.52 \\
\hline Carcoforo $^{22}$ & 2006 & 566 & 26 (median) & 3 & 0.53 \\
\hline Takei $^{16}$ & 2007 & 1,062 & 34 (median) & 4 & 0.38 \\
\hline Bergkvist $^{15}$ & 2008 & 2,246 & 37 (median) & 27 & 1.20 \\
\hline Poletti ${ }^{18}$ & 2008 & 804 & 38.8 (median) & 6 & 0.75 \\
\hline Kiluk & 2009 & 1,530 & 63 (median) 59.1 (mean) & 4 & 0.26 \\
\hline
\end{tabular}

greater than the historical values of recurrence following axillary dissection. As Naik et al. reviewed, in clinically node-negative patients who undergo axillary dissection, axillary recurrence rates are less than $2 \%$ with greater than 5-year follow-up. ${ }^{14,24-27}$ Furthermore, recurrence rates with either sentinel node biopsy or axillary dissection compare favorably with axillary recurrence rates without axillary treatment. For T1c and T2 lesions, local axillary recurrence rates have been found to be $10 \%$ and $18 \%$, respectively, with 5 years of follow-up when the axilla is not addressed by either axillary dissection, sentinel node biopsy, or axillary radiation. ${ }^{28}$

As surgeons, it must be clearly stated that the role of surgery in the treatment of breast cancer is twofold: accurate staging and local control. The status of the axilla in breast cancer plays an important role in determining prognosis as well as maximizing local axillary control. While not the focus of this paper, the improved accuracy for breast cancer staging of sentinel node mapping achieves the primary surgical objective. This study demonstrates that the surgical role of regional control with a mean of 5 years of follow-up in over 1,000 patients is not compromised by avoiding axillary dissection in patients with negative sentinel lymph node biopsy.

ACKNOWLEDGMENT We thank Jeff King at the H. Lee Moffitt Cancer Center Comprehensive Breast Cancer Program Database and Helen Lewis at the Cancer Registry for their assistance in data retrieval. We also thank Samira Y. Khera, MD, Danielle M. Hasson, Laura B. White, and Elisabeth L. Dupont, MD for their assistance.

\section{REFERENCES}

1. Fisher B, Slack NH. Number of lymph nodes examined and the prognosis of breast carcinoma. Surg Gynecol Obstet. 1970;131(1):79-88.

2. Nemoto T, et al. Management and survival of female breast cancer: results of a national survey by the American College of Surgeons. Cancer. 1980;45(12):2917-24.
3. Petrek JA, PI Pressman, RA Smith. Lymphedema: current issues in research and management. CA Cancer J Clin. 2000;50(5):292307.

4. Bass SS, et al. Lymphatic mapping and sentinel lymph node biopsy. Breast J. 1999;5(5):288-95.

5. Fleissig, A, et al. Post-operative arm morbidity and quality of life. Results of the ALMANAC randomised trial comparing sentinel node biopsy with standard axillary treatment in the management of patients with early breast cancer. Breast Cancer Res Treat. 2006;95(3):279-93.

6. Lucci, A, et al. Surgical complications associated with sentinel lymph node dissection (SLND) plus axillary lymph node dissection compared with SLND alone in the American College of Surgeons Oncology Group trial Z0011. J Clin Oncol. 2007;25(24):3657-63.

7. Krag DN, et al. Technical outcomes of sentinel-lymph-node resection and conventional axillary-lymph-node dissection in patients with clinically node-negative breast cancer: results from the NSABP B-32 randomised phase III trial. Lancet Oncol. 2007;8(10):881-8.

8. Liberman L. Pathologic analysis of sentinel lymph nodes in breast carcinoma. Cancer. 2000;88(5):971-7.

9. Veronesi U, et al. A randomized comparison of sentinel-node biopsy with routine axillary dissection in breast cancer. $N$ Engl J Med. 2003;349(6):546-53.

10. Marrazzo A, et al. Is sentinel lymph node biopsy more accurate than axillary dissection for staging nodal involvement in breast cancer patients? Chir Ital. 2007;59(5):693-9.

11. Cox CE. Lymphatic mapping in breast cancer: combination technique. Ann Surg Oncol. 2001;8(9 Suppl):67S-70S.

12. Bass SS, et al. The role of sentinel lymph node biopsy in breast cancer. J Am Coll Surg. 1999;189(2):183-94.

13. van der Ploeg IMC, et al. Axillary recurrence after a tumournegative sentinel node biopsy in breast cancer patients: a systematic review and meta-analysis of the literature. Eur J Surg Oncol. 2008;34(12):1277-84.

14. Naik A, et al. The risk of axillary relapse after sentinel lymph node biopsy for breast cancer is comparable with that of axillary lymph node dissection: a follow-up study of 4008 procedures. Ann Surg. 2004;240(3):468-71.

15. Bergkvist $\mathrm{L}$, et al. axillary recurrence rate after negative sentinel node biopsy in breast cancer: three-year follow-up of the Swedish Multicenter Cohort Study. Ann Surg. 2008;247(1):150-6. doi: 10.1097/SLA.0b013e318153ff40.

16. Takei H, et al. Recurrence after sentinel lymph node biopsy with or without axillary lymph node dissection in patients with breast cancer. Breast Cancer. 2007;14(1):16-24. 
17. Veronesi, U, et al. Sentinel node biopsy in breast cancer: early results in 953 patients with negative sentinel node biopsy and no axillary dissection. Eur J Cancer. 2005;41(2):231-7.

18. Poletti $\mathrm{P}$, et al. Axillary recurrence in sentinel lymph node-negative breast cancer patients. Ann Oncol. 2008;19(11):1842-6.

19. Blanchard DK, et al. Relapse and morbidity in patients undergoing sentinel lymph node biopsy alone or with axillary dissection for breast cancer. Arch Surg. 2003;138(5):482-7; discussion 487-8.

20. Jeruss JS, et al. Axillary recurrence after sentinel node biopsy. Ann Surg Oncol. 2005;12(1):34-40.

21. Swenson KK, et al. Axillary disease recurrence after sentinel lymph node dissection for breast carcinoma. Cancer. 2005;104(9):1834-9.

22. Carcoforo P, et al. Accuracy and reliability of sentinel node biopsy in patients with breast cancer. Single centre study with long term follow-up. Breast Cancer Res Treat. 2006;95(2):111-6.

23. de Kanter AY, et al. 5-Year follow-up of sentinel node negative breast cancer patients. Eur J Surg Oncol. 2006;32(3):282-6.
24. Veronesi U, et al. Conservative treatment of early breast cancer. Long-term results of 1232 cases treated with quadrantectomy, axillary dissection, and radiotherapy. Ann Surg. 1990;211(3): 250-9.

25. Recht A, et al. Regional nodal failure after conservative surgery and radiotherapy for early-stage breast carcinoma. J Clin Oncol. 1991;9(6):988-96.

26. Chua B, Ung O, Boyages J. Competing considerations in regional nodal treatment for early breast cancer. Breast J. 2002;8(1):1522.

27. Louis-Sylvestre C, et al. Axillary treatment in conservative management of operable breast cancer: dissection or radiotherapy? Results of a randomized study with 15 years of follow-up. J Clin Oncol. 2004;22(1):97-101.

28. Greco M, et al. Breast cancer patients treated without axillary surgery: clinical implications and biologic analysis. Ann Surg. 2000;232(1):1-7. 